\title{
Improvement in Sperm DNA Quality Following Simple Life Style Intervention: A Study in Fathers of Children with Non-Familial Sporadic Heritable Retinoblastoma
}

\author{
Shiv Basant Kumar ${ }^{1}$, Surabhi Gautam¹, Madhuri Tolahunase¹, Bhavna Chawla², Raj Kumar Yadav³, Prabin Kumar ${ }^{4}$, D K Mitra ${ }^{4}$ and Rima \\ Dada $^{1 *}$ \\ ${ }^{1}$ Department of Anatomy, Laboratory for molecular reproduction and genetics, All India Institute of Medical Sciences, New Delhi, India \\ ${ }^{2}$ Department of Ocular Oncology and Pediatric Ophthalmology Service, Rajendra Prasad Centre for Ophthalmic Sciences, India \\ ${ }^{3}$ Department of Physiology, Integral Health Clinic, All India Institute of Medical Sciences, New Delhi, India \\ ${ }^{4}$ Department of HLA and Immunogenetics, AlIMS, New Delhi, India
}

\begin{abstract}
Introduction and objectives: Genomic integrity of gametes is important for birth of healthy offspring. As compared to ova the sperm is more susceptible to mutation due to limited DNA damage detection and repair mechanism. Life style habits adopted by father before conception may adversely affect genomic DNA in male gametes. Oxidative stress damages sperm nuclear and mitochondrial DNA. Complementary and alternative medical therapies such as yoga/meditation are being increasingly used as adjuncts to modern medicine in treatment of several clinical conditions. Therefore, in this pilot study, we analyzed the effects of yoga and meditation on seminal oxidative stress and sperm DNA integrity in fathers of children with non-familial sporadic heritable retinoblastoma $(\mathrm{Rb})$ after intervention (3 and 6 months).

Methods: A total of 10 men (father of children with sporadic non-familial $\mathrm{Rb}$ ) were recruited in this study. Semen samples were collected at base line (day 0), 3 months and after 6 months of yoga practice. Reactive oxygen species (ROS), DNA fragmentation index (DFI) and 8-hydroxy-2'-deoxyguanosine (8-OHdG) levels were estimated at day 0, 3 months and 6 months interval.

Results: There was reduction in mean DFI levels [p>0.05] at 3 months and 6 months [ $p<0.05]$ compared to base level (day 0). Seminal mean ROS levels were significantly reduced after 3 months [p<0.05] and 6 months [p<0.01] We also observed reduction in levels of $8-O H d G$ both after 3 months [p>0.05] 6 months [p<0.05] with respect to the base line levels (day 0)

Conclusions: Yoga/meditation may significantly lower oxidative stress and oxidative nuclear DNA damage and levels of the mutagenic base $8 \mathrm{OHdG}$. Thus yoga and meditation are simple life style interventions which are therapeutic for maintaining/restoring sperm DNA integrity. Recently United Nation's proposal to celebrate International Yoga Day (21 st June) might be an encouraging step
\end{abstract}

Keywords: Retinoblastoma; SCSA; DFI, ROS; Sperm DNA damage; Yoga; Meditation

\section{Introduction}

Although yoga has been practiced for over 5,000 years, it has only recently gained popularity in the United States, Europe and other parts of world. Research into the role of yoga in certain disease states and in improving overall health is ongoing. Recent studies have shown that sperm with oxidative damage are associated with increased rates of mutagenesis and carcinogenesis since sperm being transcriptionally and translationally inert and have a very basic repair mechanism against such damage $[1,2]$. Sperm mitochondrial and nuclear DNA are specifically prone to oxidative damage due to lack of an efficient repair system [2]. This extensive damage to sperm DNA or aging oocyte may overwhelm the oocyte repair mechanism and thus result in maintenance of DNA damage in genomic DNA. This may be the common underlying etiology of infertility, recurrent spontaneous abortion, congenital malformation and even childhood cancer [3].

Factors such as an erratic lifestyle including poor diet, sedentary life style, substance abuse, and psychological stress have been shown to inflict oxidative DNA damage to sperm [4]. Such damage may result in increased DFI, and accelerated telomere shortening compromising the quality of sperm [5]. The key evidence comes from in-vitro fertilization studies, which show that there is an increased incidence of imprinting disorders in these children [6]. Recent studies have also shown an increase in autosomal dominant disorders, several cancers in children of fathers who smoke [7]. Therefore, it is plausible that a healthy lifestyle incorporating meditation and yoga might lower the seminal oxidative stress might lower the stress levels and improve DNA integrity in sperm.

Studies have shown that a short-term yoga-based lifestyle intervention reduces stress and inflammation and oxidative damage [79]. Therefore we hypothesize that regular practice of yoga/meditation may reduce seminal free radical levels and oxidative damage in the sperm. This preliminary study highlights the role of yoga and meditation in maintaining the genomic integrity of germ cells for birth of healthy offspring and there by reduce the incidence of childhood morbidity and even cancer.

*Corresponding author: Rima Dada, Department of Anatomy, Laboratory for mo lecular reproduction and genetics, All India Institute of Medical Sciences, New Delhi, India, Tel: 0112658 8700; E-mail: rima_dada@rediffmail.com

Received January 27, 2015; Accepted March 27, 2015; Published March 29 2015

Citation: Kumar SB, Gautam S, Tolahunase M, Chawla B, Yadav RK, et al. (2015) Improvement in Sperm DNA Quality Following Simple Life Style Intervention: A Study in Fathers of Children with Non-Familial Sporadic Heritable Retinoblastoma. J Clin Case Rep 5: 509. doi:10.4172/2165-7920.1000509

Copyright: (c) 2015 Kumar SB, et al. This is an open-access article distributed under the terms of the Creative Commons Attribution License, which permits unrestricted use, distribution, and reproduction in any medium, provided the original author and source are credited. 


\section{Materials and Methods}

\section{Study design and subjects}

In this study 31 fathers of children with Rb reporting at outpatient department at All India Institute of Medical Sciences, New Delhi but majority of subjects were excluded because parents harbored somatic mutations. Total of 10 men were included which participated and complied with the regular practice of yoga and meditation. A structured questionnaire was used to obtain information on demographic characteristics, birth-related factors, childhood medications, residential history, and familial and genetic factors, as well as on maternal and paternal medications, occupation, and other environmental and lifestyle factors, such as smoking and alcohol use prior to and following the conception of the baby. Other confounding factors (varicocele, history of antioxidant intake etc.) that may affect DNA damage levels were ruled out and such cases were excluded from study.

The study was approved by Institutional Ethics Committee (IESC/T-364), and the subjects provided written informed consent prior to any study specific procedures.

To reduce their stress as caregivers, the subjects underwent a 2 week supervised yoga-based lifestyle interventions per our advice, and were followed-up for up to 6 months. The study assessments were done pre and post intervention at Baseline, after 3 months and 6 months.

\section{Yoga-based lifestyle intervention}

The supervised yoga-based intervention is a regular outpatientbased program, practiced for two hours each day for the 2 weeks. The participants attend the Integral Health Clinic (IHC), Department of Physiology, All India Institute of Medical Sciences, New Delhi. The intervention consists of an integrated and pretested yoga-based intervention comprising of theory and practice sessions [8,9]. A typical day in the program starts with a set of simple physical postures (asanas) and breathing exercises (pranayama) for approximately one hour. This is followed by an interactive lecture/ discussion on stress management, importance of yoga in life, nutrition etc. Each day's program ends with relaxation through either a relaxation technique (shavasana) or meditation. Spouse and other members of the participant's family are motivated to attend the program in order to facilitate compliance and there is regular counseling and follow up of each case.

Thereafter, participants were advised to follow the same for up to 6 months. During this period, participants were followed-up at least once a week for compliance, and were also advised to visit the IHC as and when required.

\section{Laboratory assessments}

Semen samples were collected by masturbation in a sterile urine culture vial after 4 days of sexual abstinence, and were analyzed for ROS and DFI on the same day. After liquefaction at $37^{\circ} \mathrm{C}$, semen analysis was performed as per the WHO guidelines [10].

\section{Sperm Chromatin structure assay}

The sperm chromatin structure assay (SCSA) was performed as per the protocol described by Evenson et al. [11]. After complete analysis of the sample, the X-mean (red fluorescence) and Y-mean (green fluorescence) values were recorded manually after selecting gate for sperm cells using FlowJO software (Oregon). Extent of DNA denaturation (damage) was expressed as DFI, which is the ratio of red to total (red plus green) fluorescence, i.e., the level of denatured DNA over the total DNA [12]. The DFI of the patients and controls were analyzed in duplicate by a single operator, and the mean value was used for comparison.

\section{Estimation of reactive oxygen species}

After liquefaction, $400 \mu \mathrm{l}$ of neat semen was used to measure ROS levels. Ten micro litters of luminol (5-amino-2, 3,-dihydro1,4-phthalazinedione, Sigma, USA) in dimethyl sulfoxide (DMSO) was added as a probe $(5 \mathrm{mM})$. A negative control was prepared by adding $10 \mu \mathrm{L}$ of $5 \mathrm{mM}$ luminol to $400 \mu \mathrm{L}$ of phosphate buffer saline. Levels of ROS were assessed by measuring the luminol-dependant chemiluminescence with the luminometer (Sirius, Berthold Detection Systems GmbH, Pforzheim, Germany) in the integrated mode for 10 minutes. The results were expressed as RLU/sec $/ 10^{6} \mathrm{sperm}$. Each sample was analyzed in duplicate and the mean was taken.

\section{8-Hydroxy-2'-deoxyguanosine assay:}

8-OHdG was measured using ELİSA (Cayman Chemical, USA) as per manufacturer's protocol. The test utilizes an anti-mouse IgG-coated plate and a tracer consisting of an 8-OHdG conjugate. This format has the advantage of providing low variability and increased sensitivity compared with assays that utilize an antigen-coated plate. The procedure followed was as described in the manufacturer's protocol. Absorbance was measured at $450 \mathrm{~nm}$ using a micro plate reader (Biotech, USA). The results were calculated with the Cayman data analysis system [13].

\section{Statistical Analysis}

The paired sample t-tests (two-tailed) was used to compare baseline values (Day 10) to those obtained at 3 months and 6 months after the intervention, $\mathrm{p}<0.05$ was used as the level of significance.

\section{Results}

The mean age of fathers was $33.8 \pm 7.16$ years. The mean BMIs were within normal range $(23.1 \pm 2.36)$. The semen parameters of cases were within the reference range, and comparable before and after intervention. The SCSA parameters, that is, DNA fragmentation index (\%DFI), indicate the fraction of defective spermatozoa (Figure1) showing the presence of DNA breaks (only two cases shown) and pattern of decrease in \% DFI post intervention. Out of 10 subjects, in nine subjects we observed decline in the DFI levels after 3 months while after 6 months of intervention 8 subjects DFI levels were reduced. At baseline (day 0$)$, the mean DFI was significantly higher $(36.22 \pm 1.13$ $\%$ DFI; $\mathrm{p}<0.001)$ than cut-off levels established in our laboratory $(22.2$ $\pm 4.13 \%$ DFI) [7]. Mean DFI reduced by about $14.38 \%$ (31.35 $\pm 2.01 \%$ DFI; $p>0.05$ ) in subjects from baseline to Month 3, and by about $29.12 \%$ $(24.7 \pm 2.91 \% \mathrm{DFI} ; \mathrm{p}<0.05)$ in subjects at compared to base line Vs Month 6 of yoga/meditation practice, which was nearly in normal range. The subjects were not on any drug/antioxidants.

The seminal mean ROS levels were significantly higher (83.91 \pm 17.72 RLU/s/million sperm; $\mathrm{p}<0.01$ ) at base level (day 0) which was significantly higher than the cut-off value established in our laboratory $(27.70 \pm 3.73 \mathrm{RLU} / \mathrm{s} / \mathrm{million}$ sperm) [7]. Following the yoga-based lifestyle intervention, the levels reduced to nearly $40 \%$ of baseline ( 51.11 $\pm 5.49 \mathrm{RLU} / \mathrm{s} / \mathrm{million}$ sperm; $\mathrm{p}<0.05$ ) at 3 months, and $68 \%$ of baseline $(26.97 \pm 6.24 \mathrm{RLU} / \mathrm{s} / \mathrm{million}$ sperm; $\mathrm{p}<0.01)$ at the end of 6 months.

The mean levels of oxidative mutagenic base, 8-hydroxy-2'deoxyguanosine (8-OHdG) was significantly higher $(90.33 \pm 7.32 \mathrm{pg} / \mathrm{ml}$; $\mathrm{p}<0.01)$ as compared to our cut-off $(33.25 \pm 6.23 \mathrm{pg} / \mathrm{ml})$ in the subject's Sperm DNA [7]. We also observed $25 \%$ reduction in mean levels of 8-OHdG after 3 months [68.22 $\pm 14.61 ; \mathrm{p}>0.05]$ and after follow up of 


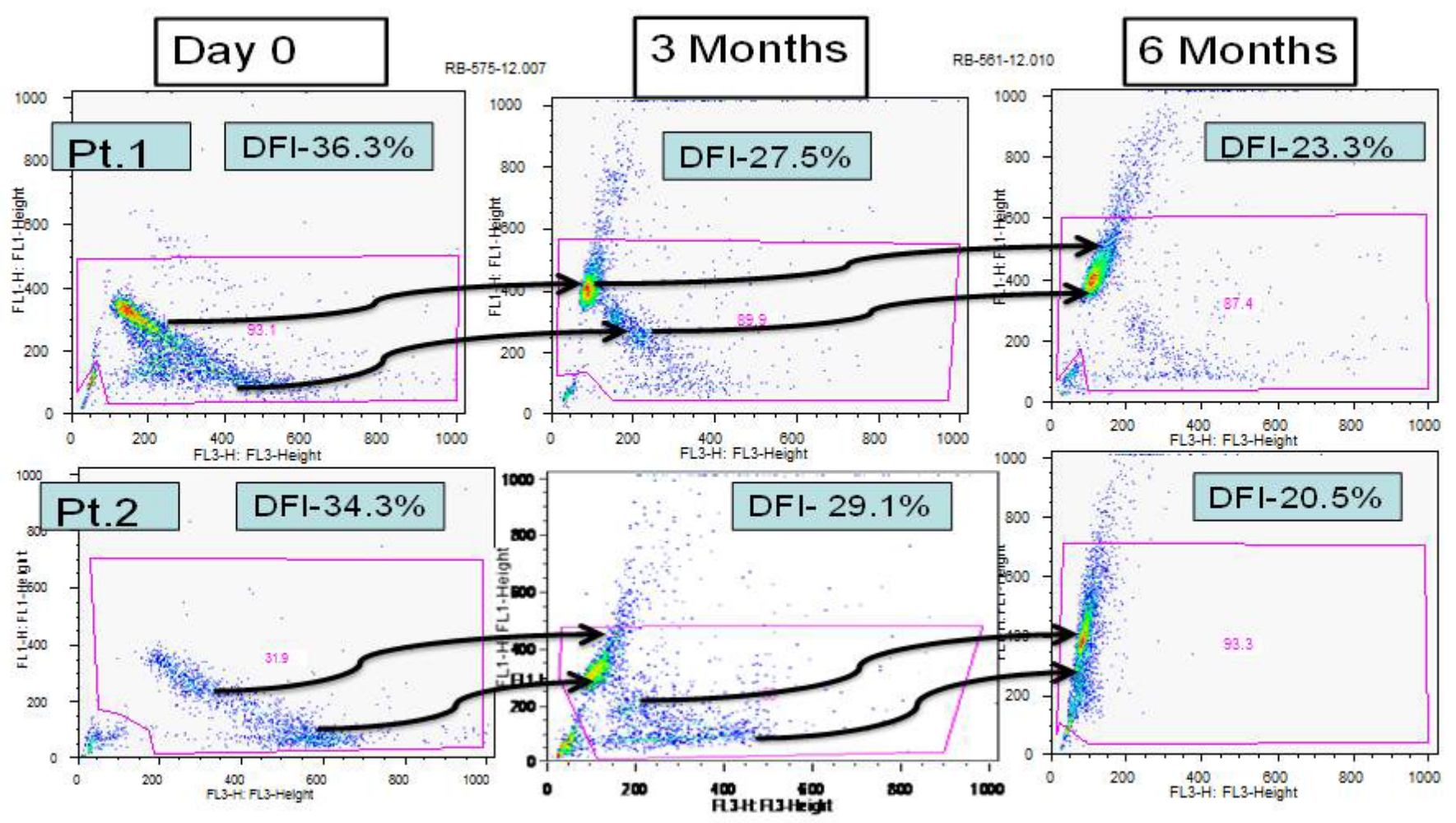

Figure 1: Dot plot cytograms of semen samples at day 0,3 months and 6 months of yoga respectively by SCSA. X-axis (FL3) represents fragmented DNA and Y-axis (FL1) represents native DNA showing (DFI \%) the percentage of spermatozoa with high levels of DNA fragmentation. Each cytogram dot represents a single spermatozoon with dual-parameter green and red fluorescence values. The debris (bottom-left corner) was excluded from the analysis.

\begin{tabular}{|c|c|c|c|c|c|c|}
\hline Assay $(\mathrm{N}=10)$ & Cut off Value [7] & $\begin{array}{c}\text { Mean } \pm \text { S.D } \\
(0-\text { Day })\end{array}$ & $\begin{array}{l}\text { Mean } \pm \text { S.D } \\
\text { (3 months) }\end{array}$ & $\begin{array}{l}\text { Mean } \pm \text { S.D } \\
\text { (6 months) }\end{array}$ & $\begin{array}{c}\text { p-Value } \\
0 \text { vs } 3 \text { months }\end{array}$ & $\begin{array}{c}\text { p-Value } \\
0 \text { vs } 6 \text { months }\end{array}$ \\
\hline DFI (\%) & $22.2 \pm 4.13$ & $36.22 \pm 1.13$ & $31.35 \pm 2.01$ & $24.7 \pm 2.91$ & $p>0.05$ & $p<0.05$ \\
\hline ROS (RLU/min/104 Neutrophils) & $27.70 \pm 3.73$ & $83.91 \pm 17.72$ & $51.11 \pm 5.49$ & $26.97 \pm 6.24$ & $p<0.05$ & $p<0.01$ \\
\hline 8-OHdG (pg/ml) & $33.25 \pm 6.23$ & $90.33 \pm 7.32$ & $68.22 \pm 14.61$ & $49.66 \pm 4.11$ & $p>0.05$ & $p<0.05$ \\
\hline
\end{tabular}

${ }^{*} \mathrm{P}$-value $<0.05$ was considered to be significant

Table 1: Comparison of the various parameters in the study groups pre and post intervention.

6 months there was $45 \%$ reduction in mean levels of 8 -OHdG [49.66 \pm 4.11; $\mathrm{p}<0.05$ ] with respect to the base level (Table 1 ).

\section{Discussion}

The data from this study show that the fathers had a highe level of ROS and raised DFI vs. established cutoff values at our lab. A yoga/ meidtation-based lifestyle intervention gradually reduced the oxidative damage in semen, and sperm over a period of 6 months. There was no improvement in DFI levels 10 days post intervention (data not shown). This is a clinically important finding since men having children with retinoblastoma might have an ongoing oxidative stress damaging the sperm DNA, and hence at an increased risk of having subsequent children with genetic disorders/ congenital malformation and even cancer.

Oxidative stress plays a major role in the etiology of various types of congenital abnormalities, and abnormal spermatozoa frequently display typical features of oxidative stress i.e. excessive level of ROS and depleted antioxidant capacity [14]. This may result in initiation of apoptosis and mutations resulting in blastocyst arrest, miscarriage, abnormalities in the offspring and an increased susceptibility to childhood cancer, and also infertility as shown by our group previously [15]. Importantly, in this study the parents had no somatic mutation in retinoblastoma gene suggesting that the mutation may have occurred in sperm DNA or very early in zygote due to heavy mutagenic load in sperm DNA as suggested by elevated levels of 8-hydroxy-2'-deoxyguanosine The seminal ROS levels declined even 10 days post intervention(data not shown)but DFI level showed no decline, at 3 months the decline was not significant and minimum 6 months of intervention is required to see the significant changes in DNA integrity. The DFI reduced gradually over a period of 6 months, along with a decrease in seminal ROS levels indicating improved functionality as well as quality of the sperm. This finding is highly relevant because though genetic causes are irreversible but oxidative DNA damage can be minimized by adopting healthy lifestyle and including daily practice of yoga and meditation [16,17]. Two cases did not show any improvement in DFI though showed decline ROS levels indicate that DNA damage in such cases may be due to other factor like defective packaging of DNA, altered protamine/histone ratio. This study also has clinical implications in cases with malefactor infertility, opting for assisted conception and may reduce pre and post implantation losses with improvement in sperm DNA quality. 
Citation: Kumar SB, Gautam S, Tolahunase M, Chawla B, Yadav RK, et al. (2015) Improvement in Sperm DNA Quality Following Simple Life Style Intervention: A Study in Fathers of Children with Non-Familial Sporadic Heritable Retinoblastoma. J Clin Case Rep 5: 509. doi:10.4172/21657920.1000509

Page 4 of 4

Overall, the results suggest that this meditation/yoga-based lifestyle intervention might result in lower oxidative stress, reduce DFI and lower mutagenic load in sperm DNA. Regular practice of yoga and meditation could also be the key to healthy senescence as it could have a buffering effect on age-dependent DNA damage and repair capacity. This study paves the road map for designing more robust studies using these variables. To the best of our knowledge, this is the first study to assess the effects of meditation/yoga on oxidative DNA damage load. This may assist the couples in planning the subsequent pregnancy, while delaying the conception till ROS levels and DNA damage decline significantly, thus lowering the incidence of childhood morbidity and cancer in offspring.

\section{References}

1. Aitken RJ, Smith TB, Jobling MS, Baker MA, De luliis GN (2014) Oxidative stress and male reproductive health. Asian J Androl 16: 31-38.

2. Koppers AJ, Garg ML, Aitken RJ (2010) Stimulation of mitochondrial reactive oxygen species production by unesterified, unsaturated fatty acids in defective human spermatozoa. Free Radic Biol Med 48: 112-119.

3. Kumar K, Deka D, Singh A, Mitra DK, Vanitha BR, et al. (2012) Predictive value of DNA integrity analysis in idiopathic recurrent pregnancy loss following spontaneous conception. J Assist Reprod Genet 29: 861-867.

4. Omu AE (2013) Sperm parameters: paradigmatic index of good health and longevity. Med Princ Pract 22 Suppl 1: 30-42.

5. Blackburn EH (2001) Switching and signaling at the telomere. Cell 106: 661673.

6. Sutcliffe AG, Peters CJ, Bowdin S, Temple K, Reardon W, et al. (2006) Assisted reproductive therapies and imprinting disorders--a preliminary British survey. Hum Reprod 21: 1009-1011.
7. Kumar SB, Yadav R, Yadav RK, Tolahunase M, Mishra SS, et al. (2014) Impact of life style interventions on markers of cellular aging. Andrology Suppl 3: 73 .

8. Yadav RK, Magan D, Mehta N, Sharma R, Mahapatra SC, et al. (2012) Efficacy of a short-term yoga-based lifestyle intervention in reducing stress and inflammation: preliminary results. J Altern Complement Med 8: 662-667.

9. Yadav RK, Ray RB, Vempati R, Bijlani RL (2005) Effect of a comprehensive yoga-based lifestyle modification program on lipid peroxidation. Indian $\mathrm{J}$ Physiol Pharmacol 49: 358-362.

10. WHO (1999) Laboratory Manual for the Examination of Human Semen and Sperm-Cervicalmucus Interaction. Cambridge University Press, UK.

11. Evenson DP, Jost LK, Marshall D, Zinaman MJ, Clegg E, et al. (1999) Utility of the sperm chromatin structure assay as a diagnostic and prognostic tool in the human fertility clinic. Hum Reprod 14: 1039-1049.

12. Evenson DP, Larson KL, Jost LK (2002) Sperm chromatin structure assay: its clinical use for detecting sperm DNA fragmentation in male infertility and comparisons with other techniques. J Androl 23: 25-43.

13. Sweta SM, Kranthi V, Kumar R, Malhotra N, Mohanty K (2014) Oxidative Damage to Sperm DNA: Clinical Implications. Andrology 3:116.

14. Aitken RJ, Koppers AJ (2011) Apoptosis and DNA damage in human spermatozoa. Asian J Androl 13: 36-42.

15. Venkatesh S, Singh A, Shamsi MB, Thilagavathi J, Kumar R, et al. (2011) Clinical significance of sperm DNA damage threshold value in the assessment of male infertility. Reprod Sci 18: 1005-1013.

16. Furness DL, Dekker GA, Roberts CT (2011) DNA damage and health in pregnancy. J Reprod Immunol 89: 153-162.

17. Agarwal A, Prabakaran SA (2005) Mechanism, measurement, and prevention of oxidative stress in male reproductive physiology. J Exp Biol 43: 963-974. 